\title{
Job Insecurity and Depressive Symptoms in Mothers and Adolescents: A Dyadic Study
}

\author{
Saija Mauno ${ }^{1,2} \cdot$ Jaana Minkkinen $\mathbb{1}^{1,2} \cdot$ Riikka Hirvonen $^{3} \cdot$ Noona Kiuru $^{2}$
}

Accepted: 31 May 2021 / Published online: 16 June 2021

(c) The Author(s) 2021

\begin{abstract}
In this study, we examined whether maternal perceived job insecurity (JI) affected depressive symptoms in their adolescent children. Specifically, we tested a mediator-moderator model in which we hypothesized that maternal JI was related to adolescent depressive symptoms indirectly via maternal depressive symptoms. Most importantly, we also explored whether this indirect path was moderated by two buffering resources, i.e., maternal off-job recovery and adolescent self-esteem. There is little research on the effects of parental JI on adolescent children from the viewpoint of buffering moderators. Due to growing insecurity in the labor markets we need more information about resources that mitigate the harmful effects of JI within families. We tested the model via structural equation modeling among Finnish mother-adolescent dyads $(N=601$, mothers aged 32-60 years; adolescents aged 14-18 years). Our results showed that mothers' depressive symptoms did not mediate the relationship between mothers' JI and adolescents' depressive symptoms, but mothers' JI was directly related to increased depressive symptoms among both adolescents and their mothers. Mothers' off-job recovery and adolescents' selfesteem buffered against mothers' JI in relation to depressive symptoms. When off-job recovery (in mothers) and self-esteem (in adolescents) were high, the higher level of JI was not associated with increased depressive symptoms. Off-job recovery among working mothers should be improved as it has potential to protect against JI. Moreover, adolescents' self-esteem should be boosted as it mitigates the effects of mothers' job stress (e.g., JI) on adolescents' well-being.
\end{abstract}

Keywords Job insecurity $\cdot$ Depressive symptoms $\cdot$ Dyadic study $\cdot$ Buffering resources $\cdot$ Adolescents

\section{Highlights}

- Parents' job insecurity may affect negatively their children.

- Mothers' job insecurity related to depressive symptoms among mothers and children.

- Mothers' off-job recovery mitigated the effect of job insecurity on their depressive symptoms.

- Adolescents' self-esteem mitigated the effect of mothers' job insecurity on their depressive symptoms.

- Buffering resources need more attention in exploring family-related consequences of job insecurity.

Jaana Minkkinen

Jaana.Minkkinen@tuni.fi

1 Faculty of Social Sciences (Psychology), Tampere University, Tampere, Finland

2 Faculty of Education and Psychology (Psychology), University of Jyväskylä, Jyväskylä, Finland

3 School of Applied Educational Science and Teacher Education, University of Eastern Finland, Joensuu, Finland

\section{Aim and Contributions}

The threat of job loss, that is, perceived job insecurity (JI), is one of the most common job stressors today due to rapid fluctuations in economy and organizational processes (e.g., De Witte et al., 2016; Vander Elst et al., 2014). Ongoing rapid technological acceleration due to digitalization, robotization, and artificial intelligence is also increasing job insecurity, causing erosion of occupations particularly in manual and blue-collar work (Taewoo, 2019). The detrimental effects of JI on employees' health and well-being are well documented in meta-analyses (Cheng \& Chan, 2008; De Witte et al., 2016; Jiang \& Lavaysse, 2018). However, much less is known 
about whether and how $\mathrm{JI}$ is transmitted from an employee to his/her family members, and more research has been called for on this topic (e.g., Mauno et al., 2017).

In this present study we adopted a dyadic approach to examine whether parental JI was associated with adolescent children's depressive symptoms. Focusing on adolescents' depressive symptoms as an outcome is important, as depression tends to have long-lasting negative consequences for mental health and global functioning throughout the life course, also affecting later career prospects and work ability (Fletcher, 2008; Mattila-Holappa, 2018). There is also some evidence to indicate that prevalence of adolescents' depressive symptoms has increased in the 21st century (Bor et al., 2014). In Finland, the context of our study, depression is also a major cause of work disability among younger age cohorts (Mattila-Holappa, 2018). Consequently, from the standpoint of sustainable careers, risk factors of adolescents' depression, including parental employment factors, e.g., JI, require urgent attention.

Specifically, we tested a mediator-moderator model in a dyadic design by examining whether maternal $\mathrm{JI}$ is related to more severe maternal depressive symptoms, which, in turn, would increase adolescents' depressive symptoms. Moreover, concerning this indirect relation, we also investigated mothers' off-job recovery and adolescents' selfesteem as potential buffering factors against the harmfulness of maternal JI. If JI cannot be avoided, as seems to be the case in today's global economy with fast technological progress (De Witte et al., 2016; Taewoo, 2019), it is vital to identify buffering factors that may help working parents to cope with JI and to mitigate its negative effects.

\section{Jl as a Job Stressor and Empirical Evidence of Parents' JI and Adolescent Children's Outcomes}

In psychological job stress research, perceived $J I$ refers most typically to the threat of job loss/anticipated unemployment consisting of affective (e.g., fear and worry) and cognitive (e.g., uncertainty) components, which, however, are highly correlated (Jiang \& Lavaysse, 2018; Vander Elst et al., 2014). However, affective JI has been found to be more predictive in relation to mental health outcomes (Jiang \& Lavaysse, 2018) and for this reason we focused on fear and worries related to potential job loss as an indicator of JI.

The detrimental effects of JI, particularly at an intraindividual level (i.e., among working parents), can be explained via the COR theory (Hobfoll, 1989), which argues that individuals are motivated to conserve and gain material and immaterial resources. In this light, the threat of not having a job (i.e., perceiving JI) means a threat of additional resource loss(es), thus threatening the conservation or gaining of other resources, for example, incomes, health/well-being and identity, which typically come along with employment (Cheng \& Chan, 2008; De Witte et al., 2016; Jiang \& Lavaysse, 2018; Mauno et al., 2017). Thus, a threat of losing a job, as a potential resource loss, may also cause increased depressive symptoms or other health issues for an employee in the form of secondary resource losses (De Witte et al., 2016; Kim \& von dem Knesebeck, 2016; Mauno et al., 2017). On the basis of this reasoning, we consider JI as a job stressor that can be assumed to cause depressive symptoms among working mothers. Previous research supports this stress-perspective at it has shown that JI is associated with various negative health outcomes, including depressive symptoms (Cheng \& Chan, 2008; De Witte et al., 2016; Jiang \& Lavaysse, 2018; Kim \& von dem Knesebeck, 2016).

Furthermore, JI along with the anticipation of job loss may be particularly stressful for working parents who are responsible for ensuring steady income and resources for the family (see Kachi et al., 2018; Mauno et al., 2017). The negative effects of JI in the family domain can be explained, e.g., via the Family Stress Model (Conger et al., 2010; Lee et al., 2013). Parents' JI may be reflected in adjustment and well-being of their adolescent children, which is one notable negative consequence of JI, thus showing the harmful interindividual effects of JI within the families (see Mauno et al., 2017). Specifically, the Family Stress Model (Conger et al., 2010) suggests that family economic hardship and pressures, which here are assumed to be associated with JI, may affect children's adjustment and well-being indirectly via the behavior and emotional functioning of the parents. When economic stress (here in the form of JI) is present, parents may feel more emotional distress, e.g., depression and anxiety, which may have negative implications for their parenting behavior (see Conger et al., 2010; Lee et al., 2013). For example, distressed parents may exhibit less positive parenting behavior, warmth, and support for their children, which then have negative reflections on their wellbeing, giving rise to, e.g., elevated risks for depressive symptoms in children (see Chapman et al., 2016; Conger et al., 2010). Furthermore, it is also possible that adolescent children hear or "sense" their parents' job-related worries due to JI, which then may cause their depressive symptoms. In this study, we propose that parents' increased distress, manifesting in parents' elevated depressive symptoms, mediates the negative relationships between economic hardship, that is, JI, between parents and adolescent children.

There is also some empirical evidence showing that JI transmits from parents to children (for a review, see Mauno et al., 2017). Earlier studies applying a dyadic design have found that parental JI predicted depressive symptoms (Barling \& Mendelson, 1999), lower self-efficacy (Lim \& Loo, 2003), and poorer school performance (Barling \& 
Mendelson, 1999; Barling et al., 1999) in children. Some studies have moreover suggested that parental JI may also have negative career-related outcomes for offspring. For example, Barling et al. (1999) found that parental JI was related to more negative work attitudes and lower work motivation reported by their children. Later Zhao et al. (2012) showed that fathers' (but not mothers') JI predicted children's lowered career self-efficacy via two genderspecific mediators: lack of support for daughters and lack of engagement in sons. Although these studies provide valuable findings on pathways between parents' JI and children's outcomes, they have often neglected potential buffering factors in this transmission process. Consequently, our study considers the possibility that mothers' (good off-job recovery) and adolescents' (high self-esteem) resources buffer against mothers' JI by mitigating its harmful effect on depressive symptoms for both mothers and adolescents. We now turn to this buffering hypothesis.

\section{Off-Job Recovery and Self-Esteem as Stress Buffers against $\mathrm{JI}$}

Stress buffers refer to various factors, often psychosocial resources, with the potential to mitigate the harmful effects of a certain stressor, here JI, on well-being and healthrelated outcomes (Rector \& Roger, 1997). Stress buffers are often relatively stable coping resources (e.g., personality factors, coping skills), which, in line with the COR theory (Hobfoll, 1989) presented above, ensure an individual's stress adaptation by allowing conservation of resources or by inhibiting subsequent resource losses. Our study considered the possibility that mothers' (effective off-job recovery) and children's (adolescents' high self-esteem) coping resources buffer against mothers' JI. Specifically, we propose that these coping resources may mitigate the harmfulness of JI among mothers and adolescents in relation to their depressive symptoms, that is, preventing both mothers and adolescents from experiencing increased depressive symptoms in the presence of high JI.

Off-job recovery (recovery) refers to replenishing the depleted resources by reversing the negative effects of job stressors and by bringing the employee to the pre-stressor level of functioning, likewise constituting a crucial coping resource preventing the accumulation of job stress and the related long-term negative health implications (Bennett et al., 2018; Meijman \& Mulder, 1998). Effective recovery also enables an employee to conserve and gain mental and physical resources needed in daily life at work and home, an idea of which is also consistent with the COR theory (Hobfoll, 1989). Recovery has been shown to be a beneficial coping resource in various demanding work settings, and for this reason we perceived it as a potential buffering factor against JI (see Bennett et al., 2018; Kinnunen et al., 2010). However, recovery has seldom been studied as a stress buffering resource against JI as only one study has shown that recovery (via mental detachment during off-job time) buffered against JI in relation to work engagement (Kinnunen et al., 2010). Thus, more evidence needs to be gathered in this respect, and here we examine whether mothers' effective recovery buffers against JI in relation to mothers' own depressive symptoms.

Another buffering resource in this study is self-esteem, a personal resource describing an individual's overall positive evaluation of the self, including both positive beliefs and emotional states in relation to the self (Rosenberg, 1979). Self-esteem affects the stress appraisal process positively, implying that individuals with higher self-esteem perceive their environment to be less stressful, show better psychological adjustment and are more stress-resilient (Rector \& Roger, 1997). Specifically, we will focus on adolescent children's self-esteem as a buffering resource as self-esteem plays a crucial role in adolescence- the life stage which we focus on (see e.g., Boden et al., 2008; Hong et al., 2016). Self-esteem may be a valuable resource in adolescence, which, in light of the COR theory (Hobfoll, 1989), should assist adolescents in conserving or gaining resources as well as helping in stress adjustment. Hence, it is reasonable to assume that high self-esteem in adolescents buffers against their mothers' negative work experiences, preventing mothers' JI from being transmitted to them. Empirical findings support the crucial role of self-esteem in adolescence as low self-esteem during adolescence has predicted greater risks of mental health problems, substance dependence, lower levels of life and relationship satisfaction, poor health, criminal behavior, and limited economic prospects during adulthood (see e.g., Boden et al., 2008). Furthermore, self-esteem has been found to buffer against depression in adolescents (Hong et al., 2016). In line with this evidence, self-esteem can be considered as one promising stress buffer, particularly in adolescence.

\section{Tested Model and Hypotheses}

Our hypotheses are based on the COR theory (explaining the relationships mainly at the intra-individual level) and the Family Stress Model (explaining the relationships mainly at the inter-individual level) described above. Our first hypothesis (H1, see Fig. 1) proposes that mothers' JI may be related to their adolescent children's higher depressive symptoms indirectly with mothers' own depressive symptoms as a mediator. Often job stressors experienced by employees affect their family members via employees' own impaired well-being (e.g., Frone, 2003; Mauno \& Kinnunen, 1999) as intra-individual well-being is the most 


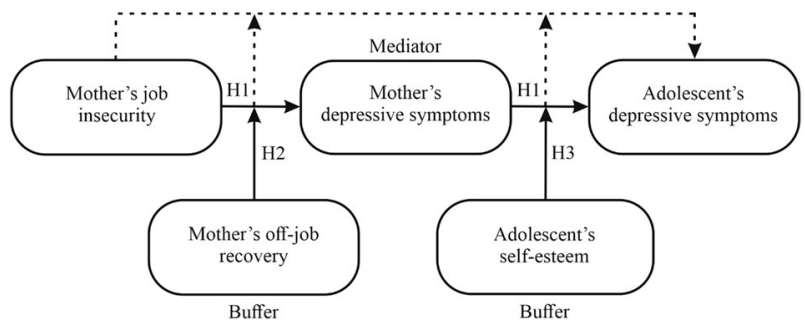

Fig. 1 A conceptual model

proximal outcome of job stressors. This idea supports the hypothesized mediator relationship (H1).

Our second hypothesis (H2, see Fig. 1) states that mothers' off-job recovery may act as a stress buffer between mothers' $\mathrm{JI}$ and their own depressive symptoms (the intra-individual buffering effect). Thus, mothers experiencing effective off-job recovery are expected to suffer less seriously from JI than mothers experiencing less effective off-job recovery and to show consequently fewer depressive symptoms.

Our third hypothesis (H3, see Fig. 1) suggests that adolescents' high self-esteem buffers against mothers' JI in relation to their depressive symptoms, but this protective pathway functions indirectly via mothers' lower depressive symptoms (the inter-individual buffering effect via a mediator). Thus, we expect a mediator-moderator model, where we hypothesize that the indirect path from mothers' JI via their depressive symptoms to adolescents' depressive symptoms is moderated by adolescents' self-esteem (Fig. $1, \mathrm{H} 3)$.

We also tested the following non-hypothetical direct relationships (without mediation); that is, adolescent's selfesteem as a buffering moderator between mother's JI and adolescent's depressive symptoms and a direct relationship between mother's JI and adolescent's depressive symptoms (see dotted lines in Fig. 1).

\section{Methods}

\section{Procedure and participants}

The present study is part of a larger research project pursuing a novel understanding of individual and environmental factors promoting schoolchildren's learning, well-being, and successful educational transitions. The project followed a community sample of over 850 adolescents through their transition from primary school to lower secondary school and further to upper secondary education in two medium-sized towns in central Finland with a combined population of 130,000. Both towns included semi-rural areas with smaller schools. The research project has the approval of the ethics committee of the local university.
The participating schools were selected with the help of local school authorities from areas where all the students transfer to particular secondary schools instead of dispersing to different locations. In one of the towns all primary and lower secondary schools were recruited, whereas in the other town the selected schools covered about $75 \%$ of the targeted age group. Before the start of the longitudinal follow-up study, all sixth-grade students and their families in the selected primary schools were contacted and $74 \%$ of the families gave their informed consent to participate in the study. When the study progressed, if new students moved from other schools and joined the participating classes, the new families were asked to participate as well. In the ninth grade, there were 13 target schools and 59 classes. In addition, 32 students from the original sample had moved to other areas but chose to continue in the follow-up. The adolescents' data were collected via questionnaires in their schools during school hours. Mothers' data were gathered via questionnaires sent to them by email or surface mail. Informed consent was provided by all participating adolescents and mothers. All participating adolescents and mothers were given a small gift token for their participation.

The final sample included 601 mother-adolescent dyads (68\% of all participating adolescents) from whom both adolescent and mother questionnaires were received. The adolescents (57\% girls) in this study were in their ninth year at comprehensive school (mean age 15.3 years; $S D=0.4$ ). The vast majority of the adolescents $(96 \%)$ reported Finnish as their first language, $2 \%$ were bilingual, and $2 \%$ had a language other than Finnish as their first language. The mothers' mean age was 46.1 years $(S D=5.4$, range $=$ 32-60 years). Information about mothers' educational level and work situation is described under control variables. A total of $75 \%$ of the families were nuclear families, $11 \%$ blended families, $13 \%$ single parents with children (either full-time or sharing custody), and $1 \%$ were some other type of families.

The sample was compared to Finnish general population in terms of mothers' educational level and family structure. In comparison with same-age women on average in Finland (Official Statistics of Finland, 2018a), the sample included fewer mothers with no vocational education (2 vs. $11 \%$ ) or vocational upper secondary education (28 vs. $39 \%$ ) and more mothers with a post-secondary college degree (21 vs. $15 \%)$, university or polytechnic university degree ( 45 vs. $34 \%$ ), or postgraduate degree (4 vs. $1 \%$ ), suggesting that the mothers in the sample were better educated than women on average in Finland. Compared to Finnish families with children under the age of 18 (Official Statistics of Finland, 2018b), two-parent families were somewhat overrepresented (86 vs. $79 \%$ ) and single-parent 
Table 1 Summary of intercorrelations, means, standard deviations and alpha-coefficients for the latent factors and control variables

\begin{tabular}{|c|c|c|c|c|c|c|c|c|c|}
\hline Measure & 1 & 2 & 3 & 4 & 5 & 6 & 7 & 8 & 9 \\
\hline 1. JI & - & & & & & & & & \\
\hline 2. Mother's off-job recovery & $-0.21 * * *$ & - & & & & & & & \\
\hline 3. Mother's depressive symptoms & $0.27 * * *$ & $-0.50 * * *$ & - & & & & & & \\
\hline 4. Adolescent's self-esteem & $-0.14 * *$ & $0.19 * * *$ & -0.10 & - & & & & & \\
\hline 5. Adolescent's depressive symptoms & $0.20 * *$ & $-0.19 * * *$ & $0.17 * *$ & $-0.79 * * *$ & - & & & & \\
\hline 6. Adolescent's gender & -0.02 & -0.01 & 0.05 & $0.32 * * *$ & $-0.29 * * *$ & - & & & \\
\hline 7. Mother's education & -0.02 & -0.01 & 0.01 & $0.10^{*}$ & -0.08 & -0.01 & - & & \\
\hline 8. Working in shifts & $0.11 * *$ & -0.04 & 0.01 & 0.09 & $-0.10^{*}$ & 0.03 & $0.31 * * *$ & - & \\
\hline 9. Type of employment contract & $-0.28 * * *$ & -0.02 & -0.03 & 0.03 & -0.07 & 0.02 & 0.00 & -0.03 & - \\
\hline$M$ & 1.55 & 2.99 & 1.76 & 3.53 & 0.65 & 0.43 & 4.51 & 0.70 & 0.86 \\
\hline$S D$ & 0.92 & 0.90 & 0.86 & 0.86 & 0.72 & 0.50 & 1.34 & 0.46 & 0.3 \\
\hline Range & $1-5$ & $1-5$ & $1-5$ & $1-5$ & $0-3$ & $0 / 1$ & $0-5$ & $0 / 1$ & $0 / 1$ \\
\hline$\alpha$ & 0.93 & - & 0.87 & 0.83 & 0.95 & - & - & - & - \\
\hline
\end{tabular}

Means, standard deviations and range base on mean variables. Correlations base on latent factors (variables 1-5) and measured control variables (variables 6-9). JI = mother's job insecurity; adolescent's gender: $0=$ girl, $1=$ boy; working in shifts: $0=$ irregular hours, $1=$ regular hours; type of employment contract: $0=$ temporary, $1=$ permanent. $\alpha=$ Cronbach's alpha-coefficient

$* p<0.05, * * p<0.01, * * * p<0.001$, two-tailed

households underrepresented (13 vs. $21 \%$ ) in the study sample.

\section{Measures}

\section{Mothers' Jl}

JI assessment was based on a global job insecurity conceptualization, referring to the subjective evaluation regarding threat of losing one's job. Specifically, we used two affectiverelated items referring to mothers' current work situation ("I'm afraid of losing my job", "I'm worried about being able to keep my job") from The Job Insecurity Scale developed and validated in Europe (Vander Elst et al., 2014). Affectiverelated items have been more predictive in relation to various psychosocial outcomes (Jiang \& Lavaysse, 2018), and for this reason we measured affective JI. The response scale was a five-point Likert scale $(1=$ completely disagree, $5=$ completely agree). Cronbach's alpha-coefficients for the JI and other sum-scales are shown in Table 1.

\section{Mothers' recovery}

Mothers' assessment of their current off-job recovery was assessed with a question ("How well have you lately recovered from work-related stress after a working day/a shift?"). Answers were given on a five-point Likert scale (1 $=$ clearly worse than usual; $5=$ clearly better than usual). Recovery has been assessed with a similar single item in earlier studies and the variable has been found to be predictive in relation to an individual's well-being/health (e.g., Niks et al., 2016).

\section{Mothers' depressive symptoms}

Mothers completed an abbreviated three-item Beck Depression Inventory (Beck et al., 1961). Items (e.g., "Lately I have felt sad") were answered on a scale ranging from 1 (not at all true of me) to 5 (very true of me) based on mothers' current situation.

\section{Adolescents' self-esteem}

Self-esteem was assessed with an abbreviated version of Rosenberg's (1965) self-esteem scale. The scale consisted of five items (e.g., "On the whole, I am satisfied with myself"; "I feel that I have a number of good qualities"), which the adolescents were asked to rate based on their prevailing feelings and thoughts about themselves using a Likert scale ranging from 1 (I totally disagree) to 5 (I totally agree).

\section{Adolescents' depressive symptoms}

Depressive symptoms were measured by using the Depression Scale (DEPS; Salokangas et al., 1995). The scale contained ten items concerning participants' depressive symptoms during the last month (e.g., "I felt sad"; "I felt that my future is hopeless"), which were answered on a 4-point scale $(0=$ not at all; $3=$ to a large extent $)$. 


\section{Control variables}

We used a few control variables to test the robustness of our mediation-moderation model. Specifically, control variables were adolescent's gender $(0=$ girl; $1=$ boy $)$, mothers' education, working in shifts $(0=$ irregular hours; $1=$ regular hours, weekdays from 8.00 to 16.00 ) for adolescent's depressive symptoms, and working in shifts and type of employment contract $(0=$ temporary; $1=$ permanent) for JI. Mothers' education was coded as follows: $0=$ no vocational education beyond comprehensive school ( $2 \%$ of mothers), $1=$ vocational upper secondary school qualification degree $(28 \%), 2=$ vocational post-secondary college qualification $(21 \%), 3=$ university of applied sciences (polytechnic) degree (18\%), $4=$ university degree $(27 \%), 5=$ university postgraduate degree; licentiate or $\mathrm{PhD}(4 \%)$.

Gender was controlled for regarding adolescent's depressive symptoms as adolescent girls tend to have depressive symptoms more often than adolescent boys (Costello et al., 2003). Mothers' education was controlled for in relation to adolescent's depressive symptoms as lower parental education has been shown to predict persistence and severity of mental disorders, including depression (McLaughlin et al., 2011). Moreover, mothers' working in shifts was also controlled for with regard to adolescent's depressive symptoms. Earlier findings on the role of shift-work for outcomes among children have been contradictory (see review Li et al., 2014). Among adolescents, mothers' working night shifts has been shown to be a risk for adolescents' depression, but irregular shifts reduced adolescents' depression due to the greater likelihood of mothers' knowing where the child was (Han \& Miller, 2009). Furthermore, we included the type of mothers' employment contract in the analyses as a control variable for JI as earlier research has shown that perceived $\mathrm{JI}$ is explained by temporary employment contract (Keim et al., 2014).

\section{Data Analysis}

Confirmatory factor analysis (CFA via Mplus software) of the five-factor measurement model was estimated using Maximum Likelihood Robust estimation. We found that the five-factor measurement model fitted the data $\left(\chi^{2}(181)=\right.$ 534.47, $p<0.001 ; \quad \mathrm{CFI}=0.95 ; \quad \mathrm{TLI}=0.94 ; \quad \mathrm{RMSEA}=$ 0.06; SRMR =0.04), suggesting that mothers' JI, mothers' recovery, mothers' depressive symptoms, adolescents' selfesteem and adolescents' depressive symptoms were separate constructs (i.e., indicating discriminant validity). The standardized estimates of factor loadings for mothers JI ranged from 0.93 to 0.94 , for mothers' recovery 0.83 , for mothers' depressive symptoms from 0.78 to 0.90 , for adolescents' self-esteem from 0.28 to 0.83 , and for adolescents' depressive symptoms from 0.57 to 0.89 . The fact that the five-factor model fitted the data well and that factor loadings were generally high suggests good construct validity and item reliability.

Second, we explored correlations between the latent factors and between the control variables and latent factors. Third, we computed structural equation modeling (SEM via Mplus software) to examine the hypothesized path from mothers' JI to adolescents' depressive symptoms via mothers' depressive symptoms without moderators. We added control variables into the SEM in the second step. We executed mediation analyses with bootstrapped $(N=1000)$ confidence intervals (MacKinnon et al., 2004).

We estimated moderation models with latent factors within the SEM framework to explore the buffering effects of both recovery and self-esteem on mediator (mothers' depressive symptoms) and outcome (adolescents' depressive symptoms) in the presence of high JI. Specifically, we computed and estimated the following interaction terms: $\mathrm{JI} \times$ recovery and $\mathrm{JI} \times$ self-esteem on mothers' and adolescents' depressive symptoms. We examined significant moderation effects further by plotting the effect of the latent independent variable on the latent dependent variable for one standard deviation above and below the mean of the latent moderator (Klein \& Moosbrugger, 2000).

It is noteworthy that in these SEM analyses we also estimated potential direct relations (between mothers' JI and adolescents' depressive symptoms) and the respective moderation effects of off-job recovery and self-esteem in this relation. These relationships were reported if they were significant. Thus, in our analytical approach all potentially meaningful associations were estimated in SEM modeling. We conducted the analyses using the statistical package of Mplus software (Version 8.3, Muthén \& Muthén, 1998-2017). We inspected the model fit for CFA and SEM by using Chi-square values $\left(\chi^{2}\right)$, comparative fit index (CFI), Tucker-Lewis index (TLI), root mean square error of approximation (RMSEA) and standardized root mean square residual (SRMR). The cutoff values used in the evaluation of model fit were 0.95 for CFI and TLI, 0.06 for RMSEA and 0.08 for SRMR (Marsh et al., 2004). We employed the missing data approach by Mplus statistical package (version 8) through full information maximum likelihood (FIML; see Muthén \& Muthén, 1998-2012). The missing data percentages in the variables were $0-15 \%$ in the analysis variables: $15 \%$ in $\mathrm{JI}, 15 \%$ in recovery from job strain, $1 \%$ in mothers' depressive symptoms, $3 \%$ in adolescents' self-esteem, $5 \%$ in adolescents' depressive symptoms, 2\% in mothers' education, $0 \%$ in adolescents' gender, $11 \%$ in the contract type, $12 \%$ in working in shifts. 


\section{Results}

\section{The Measurement Model via CFA}

We tested a measurement model with five correlated factors: mothers' JI (two items), mothers' recovery (one item), mothers' depressive symptoms (three items), adolescents' self-esteem (five items) and adolescents' depressive symptoms (ten items). The latent factor for recovery with one observed item was created by fixing the residual variance of the item at 0.25 . Residual variances of the two items of mothers' JI were fixed to be equal to avoid negative residual variances. We found that all standardized factor loadings were significant at the 0.001 level and ranged from 0.279 to 0.943 (see correlations between the factors in Table 1). Overall, the five-factor measurement model fitted the data well $\left(\chi^{2}(181)=534.47, p<0.001 ; \mathrm{CFI}=0.95\right.$; $\mathrm{TLI}=0.94$; RMSEA $=0.06$; SRMR $=0.04)$.

Furthermore, as the latent factors of adolescents' depressive symptoms and self-esteem were highly correlated ( $r=-0.79, p<0.001$; Table 1$)$, we ran two additional CFA tests for these latent variables to confirm that they were indeed separate constructs. The results showed that adolescents' depressive symptoms and self-esteem were related but separate constructs (more information about these models available from the authors).

\section{The Mediation Model via SEM: Testing HI}

The mediation model included three latent factors and their relations: mothers' JI, mothers' depressive symptoms and adolescents' depressive symptoms. We found that the fit for the model was good: $\chi^{2}(88)=303.72, p<0.001 ; \mathrm{CFI}=$ $0.97 ; \quad$ TLI $=0.96 ; \quad$ RMSEA $=0.06 ; \quad$ SRMR $=0.03$. The results showed that mothers' JI had a significant positive association with their own $\left(b^{*}=0.256, S E=0.054, p<\right.$ $0.001)$ and with their adolescents' depressive symptoms ( $b^{*}=0.144, S E=0.057, p=0.012$ ), implying that the higher mothers' JI, the more depressive symptoms in both mothers and adolescents'. However, the hypothesized mediation path (rejecting H1) from mothers' JI to adolescents' depressive symptoms via mothers' depressive symptoms was not significant (indirect effect $b^{*}=0.022$, $S E=0.014, p=0.123$ ). Thus, mothers' JI was positively related to their own depressive symptoms as well as to their adolescents' depressive symptoms, but this latter path was direct and not mediated via mothers' depressive symptoms. Explanation rates $\left(R^{2}\right)$ were $7 \%$ for mothers' and $4 \%$ for adolescents' depressive symptoms.

Including control variables (i.e., adolescents' gender, mothers' education, working in shifts and type of employment contract) in the SEM did not affect the paths reported above. The fit for the model including the control variables was also good: $\chi^{2}(143)=391.25, p<0.001 ; \mathrm{CFI}=0.96$; $\mathrm{TLI}=0.96$; RMSEA $=0.05 ; \mathrm{SRMR}=0.03$. Being a girl was the strongest predictor of adolescents' higher depressive symptoms $\left(b^{*}=-0.302, S E=0.034, p<0.001\right)$. Furthermore, we found higher depressive symptoms among adolescents whose mothers were less educated $\left(b^{*}=\right.$ $-0.086, S E=0.041, p=0.036)$ and had irregular working hours $\left(b^{*}=-0.092, S E=0.043, p=0.031\right)$. Non-shift work $\left(b^{*}=0.092, S E=0.042, p=0.030\right)$ and fixed-term employment contract $\left(b^{*}=-0.279, S E=0.057, p<0.001\right)$ predicted higher JI among the mothers. Explanation rates $\left(R^{2}\right)$ were $6 \%$ for mothers' and $15 \%$ for adolescents' depressive symptoms.

\section{The Interaction Models via SEM: Testing H2 and H3}

Specifically, we analyzed three moderation effects: JI $\times$ recovery on mothers' depressive symptoms (testing $\mathrm{H} 2$ ), $\mathrm{JI} \times$ recovery on adolescents' depressive symptoms, and $\mathrm{JI} \times$ self-esteem on adolescents' depressive symptoms. It is noteworthy that $\mathrm{H} 3$ was not testable as the mediator model, as reported above, was not supported. Specifically, we found that mothers' recovery buffered against the effect of JI on their own depressive symptoms (the interaction effect of $\mathrm{JI} \times$ recovery on mothers' depressive symptoms; $b^{*}=$ $-0.241, S E=0.68, p<0.001)$. This significant interaction relationship supported $\mathrm{H} 2$. We further examined the interaction relationship using a regression plot, which revealed that when recovery was poor (one standard deviation below the mean; see regression slope $a$ in Fig. 2), higher JI was associated with more depressive symptoms in mothers. When recovery was good (one standard deviation above the mean; see slope $b$ ), the level of depressive symptoms was under the mean in all levels of JI. Thus, effective recovery buffered against high JI by mitigating its stressfulness in relation to mothers' depressive symptoms.

\section{JOB INSECURITY AND DEPRESSIVE SYMPTOMS IN DYADS}

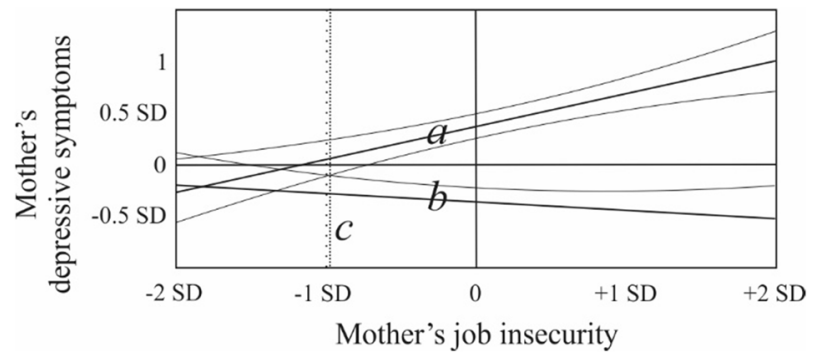

Fig. 2 Interaction of mother's recovery and JI predicting mother's depressive symptoms. Regression slope $a$ illustrates mother's depressive symptoms when recovery is poor ( $1 \mathrm{SD}$ below mean) and regression slope $b$ when recovery is good (1 SD above mean). Line $c$ illustrates the lowest level of mother's JI when the interaction is significant. Confidence intervals of $95 \%$ are presented above and below the slopes. The figure is based on standardized scales 
JOB INSECURITY AND DEPRESSIVE SYMPTOMS IN DYADS

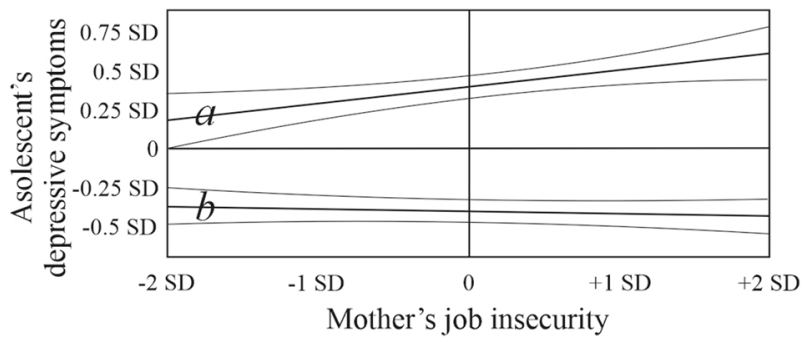

Fig. 3 Interaction of adolescent's self-esteem and JI predicting adolescent's depressive symptoms. Regression slope $a$ illustrates adolescent's depressive symptoms when adolescent's self-esteem is poor (1 SD below mean) and regression slope $b$ when adolescent's selfesteem is good (1 SD above mean). Confidence intervals of $95 \%$ are presented above and below the slopes. The figure is based on standardized scales

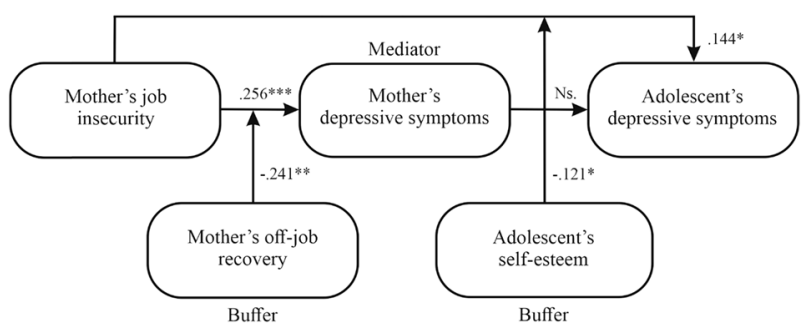

Fig. 4 A summary on significant paths. $* p<0.05$, **p $<0.01$, ***p $<$ 0.001 , two-tailed

However, in the second interaction model, mothers' recovery did not moderate the effect of her JI on adolescents' depressive symptoms (the interaction effect of $\mathrm{JI} \times$ recovery on adolescents' depressive symptoms; $b^{*}=$ $-0.073, S E=0.113, p=0.519)$. The third interaction model included adolescents' self-esteem as a buffer against the effect of mothers' JI on adolescents' depressive symptoms. We found a significant interaction effect (the interaction effect of JI $\times$ adolescents' self-esteem on adolescents' depressive symptoms; $b^{*}=-0.121, \quad S E=0.047, \quad p=$ 0.010). Mothers' higher JI was associated with more depressive symptoms in the adolescents if the adolescents' self-esteem was low (one standard deviation below the mean; see regression slope $a$, Fig. 3). When the adolescents' self-esteem was high (one standard deviation above the mean; see slope $b$ ), mothers' high JI was not associated with adolescents' depressive symptoms. Thus, adolescents' high self-esteem acted as a buffer against mothers' JI in relation to adolescents' depressive symptoms. All significant paths found in the SEM analysis are summarized in Fig. 4.

\section{Additional analyses}

We conducted additional analyses in which the effects of family structure (a proxy for marital status) and the number of children in the family were controlled for. The main pattern of the results did not change and any of the key relationships reported above remained significant.

\section{Discussion}

In this study we examined the effects of stress transmission between mothers' JI (job insecurity) and their adolescent children's depressive symptoms in Finnish mother-child dyads. Specifically, we tested a mediatormoderator model where mothers' depressive symptoms were expected to mediate the association between their JI and adolescents' depressive symptoms. Furthermore, two moderators, namely mothers' off-job recovery and adolescents' self-esteem, were hypothesized to serve as stress buffers in this mediator relationship. Whereas we found no support for the hypothesized mediator model (rejecting H1), the anticipated buffering effects were partly supported (supporting $\mathrm{H} 2$ ) in regards to direct associations between $\mathrm{JI}$ and depressive symptoms in mothers and adolescents.

\section{JI Related to Higher Depressive Symptoms both in Adolescents and Mothers}

Our results showed a significant stress-transmission relationship between mothers' JI and adolescents' depressive symptoms, indicating that the more severe mothers' JI, the more severe depressive symptoms their adolescent children reported (an inter-individual effect). This result can be explained, e.g., via the Family Stress Model (Conger et al., 2010). JI seems to be a job stressor causing economic distress for working parents and so also harmful repercussions within a family, leading to well-being problems in children. This may occur due to suboptimal parenting behavior because parents distressed by economic hardship (due to a threat of job loss) may lack the resources for optimal parenting (see Conger et al., 2010; Lee et al., 2013). However, it is also possible that children, or in this case, adolescents, observe or hear their parents' job-related worries caused by JI, which is then reflected in their well-being. This explanation, in turn, is in line with the emotion contagion model, which suggests that emotions and feelings are contagious in close relationships (Hatfield et al., 1994). Our finding is also consistent with empirical studies on the harmful effects of parental JI on children, which so far have been conducted outside the Nordic countries (e.g., Barling \& Mendelson, 1999; Mauno et al., 2017; Zhao et al., 2012). Our results showed that JI also has negative family-related implications in Scandinavia, thereby lending support to the generalizability of the findings concerning the child-related 
outcomes of JI. However, studies in this field have mostly focused on children's career-/school-related outcomes (for a review, see Mauno et al., 2017), whereas we investigated adolescent children's subjective well-being with regard to depressive symptoms as an outcome of mothers' JI. Altogether, the evidence gathered in different studies shows that parental JI may constitute a risk factor for both children's career- and well-being-related outcomes.

We also found that JI was a risk factor for working mothers' own well-being (an intra-individual effect) as it was associated with more depressive symptoms in Finnish mothers. This finding is entirely consistent with strong research evidence showing that JI has many detrimental implications for employees' well-being and health (for reviews, see Cheng \& Chan, 2008; De Witte et al., 2016), and such negative implications may actually concern more parents than non-parents (Conger et al., 2010; Kachi et al., 2018). Furthermore, this result is consistent with theories introducing JI as a job stressor, e.g., the COR theory (Hobfoll, 1989): JI threatens employees' important material and immaterial resources (e.g., income, social relationships, identity), which is distressing for them, causing detrimental health outcomes as secondary resource losses.

However, our first hypothesis (H1) on a mediator connection, which proposed a relationship between mothers' JI and adolescents' depressive symptoms via mothers' own depressive symptoms, was not supported. One possible explanation for this finding is that some other factors may better mediate the relationships between mothers' JI and adolescents' depressive symptoms. For example, mothers' external behavior (rather than internal behavior in terms of depressive symptoms) such as, irritation caused by JI, may more likely operate as a mediator in this relationship. More visible emotions may be more contagious in close relationships. We propose that future studies should investigate other possible mediator mechanisms in this respect (e.g., other emotions, parenting behavior, and parent-child interaction) that may underlie the association between parents' JI and children's (of different ages) depressive symptoms. This information would also be valuable for interventions by pinpointing the critical factors between parents' JI and children's depressive symptoms (or some other related outcomes). Another explanation for this non-existed finding (contrasting HI) might be statistical; the magnitude of zeroorder correlation between mothers' and adolescents' depressive symptoms was relatively low $(r=0.17, p<$ $0.01)$. Due to the modest strength of the association between mothers' and adolescents' depressive symptoms, it was also challenging to empirically establish a significant indirect relation after accounting for the direct effect of mother's JI and the effects of the relevant covariates.

\section{Effective Off-Job Recovery and Self-Esteem Buffered against $\mathrm{Jl}$, but Without Mediation}

Interaction analyses performed with SEM showed that mothers' successful off-job recovery and adolescents' high self-esteem functioned as buffering resources against JI. The first finding, concerning recovery as a stress buffer, supported our second hypothesis (H2; mothers' recovery acts as a stress buffer between their JI and depressive symptoms). Hence, when mothers' recovery was effective, JI did not relate to elevated depressive symptoms, but when recovery was less effective higher JI was associated with increased depressive symptoms. This finding underscores the importance of off-job recovery as an important coping resource for employees who are insecure in their jobs (Kinnunen et al., 2010). The result is also fully consistent with recent recovery research emphasizing the overall beneficial role of recovery for employees' well-being and health (see e.g., Bennett et al., 2018). Our finding is also consistent with the COR theory (Hobfoll, 1989): effective off-job recovery is a significant coping resource for working parents, helping them in stress adjustment when resources (here jobs) are threatened.

However, the finding on a buffering role of adolescents' self-esteem, did not support our third hypothesis (H3; adolescents' high self-esteem buffers against mothers' JI in relation to adolescents' depressive symptoms indirectly via lower maternal depressive symptoms). As discussed above, the hypothesized mediator-moderator model was not empirically confirmed, and therefore $\mathrm{H} 3$ was not even testable. Nevertheless, we found another interesting interaction finding showing that adolescents' high self-esteem buffered directly against mothers' $\mathrm{JI}$ in relation to adolescents' depressive symptoms. Hence, among those adolescents whose self-esteem was high, mothers' JI was not related to more severe depressive symptoms, whereas among those adolescents whose self-esteem was low mothers' JI was related to elevated depressive symptoms. Accordingly, adolescents with high self-esteem were not so likely to be affected by mothers' JI. Self-esteem is regarded as one of most remarkable personal resources (Rector \& Roger, 1997; Rosenberg, 1979), and many studies also show that lacking self-esteem in adolescence can have various negative longterm consequences, e.g., mental health problems, poorer life satisfaction, and limited economic prospects (e.g., Boden et al., 2008; Mattila-Holappa, 2018). Also this finding can be viewed in light of the COR-theory (Hobfoll, 1989): selfesteem is a valuable personal coping resource for adolescents contributing to their stress adjustment by preventing their parents' anticipated resource losses, i.e., in the form of JI, from affecting their well-being. 


\section{Limitations and Implications for Future Studies}

Our study has few noteworthy limitations. First, the design was cross-sectional, hence inhibiting the drawing of reliable conclusions, for example, on the direction of the relations between JI and depressive symptoms. However, longitudinal evidence points to the conclusion that JI predicts well-being rather than vice versa (De Witte et al., 2016). In the future, we need longitudinal and intervention studies focusing on working parents experiencing JI. Second, we collected all information using one method (survey), which may cause common method variance bias. On the other hand, using two different informants (mother-adolescent dyads) should decrease such effects. Future studies on JI in the family context should consider other than self-report measurements, e.g., in assessing parents' and children's depression in terms of use of health care services (due to mental reasons) or diagnoses.

Third, besides the other mediators (except mothers' depressive symptoms) already suggested, there may well be other relevant buffering moderators. Here, we focused only on two buffering factors, mothers' off-job recovery and adolescents' self-esteem, but there may be many other important stress buffers particularly in this context, e.g., mothers'/children's coping and self-regulation strategies. However, these buffering resources were not measured in our study, which points to the need for further research with a broader scope of moderators. A related point is that not all relevant control variables were adjusted for, and some of those could also act as moderators.

The fourth limitation is that our study was conducted in one Nordic country (Finland) and should be replicated in other contexts where working conditions and social and health insurance policies are different. For example, in Finland, the unemployed are provided with good support from the state, which may mean that JI is less stressful here than in other countries with less generous unemployment support systems. One related point is that the level of JI was low in our study $(M=1.55, S D=0.92$, scale range $1-5)$. However, despite this we were able to show that JI, also in lower levels, associated with mothers' and adolescents' depressive symptoms. Therefore, JI may constitute a harmful job stressor affecting not only working parents but also their family members (see Barling \& Mendelson, 1999; Mauno et al., 2017; Zhao et al., 2012).

The fifth limitation concerns the age of the adolescents as we studied only one age cohort (those born in 2002). Teenagers may want to distance themselves from their parents as a normative developmental process, which may mean that parents' job stressors or negative emotions are not so easily passed on, explaining partly the relatively little explained variance in this study. Thus, the effects found here may be more marked in younger children, a hypothesis that needs to be confirmed in future dyadic studies on parents' JI and its effects on their children. Furthermore, we excluded fathers from this study as their subsample was too small for a comparative design (fathers vs. mothers). However, some studies, albeit conducted in non-European contexts, propose that mothers' and fathers' JI may have different outcomes for their children (Zhao et al., 2012). Thus, fathers' possible transmission of JI to their children should be explored more in Europe.

\section{Practical Implications}

Our findings have notably practical implications stemming mostly from the buffering effects found. We recommend the development and implementation of interventions, through training, mentoring or coaching, that improve, on the one hand, off-job recovery among job-insecure parents and, on the other hand, adolescents' self-esteem. Thus, both parents and adolescent children should be considered when making recommendations for interventions.

\section{Recommendations for working parents}

Off-job recovery typically occurs via recovery experiences and recent meta-analysis indicates that psychological detachment (not thinking about work during off-job time, switching-off mentally while not working) and relaxation (having a low activation level during off-job time), are the most powerful recovery experiences predicting lower fatigue, tiredness and exhaustion (see Bennett et al., 2018). We can assume that these two recovery experiences work equally well regarding employees' depressive symptoms, which we studied as a well-being outcome. We propose that job-insecure employees would benefit from psychological detachment and relaxation during non-work time to avoid experiencing depressive symptoms or other negative effects on well-being caused by JI (see also Kinnunen et al., 2010). Recovery experiences, e.g., psychological detachment from work and relaxation, allow our mental and also our physical resources to be conserved and to recover during off-job time (see Meijman \& Mulder, 1998, Niks et al., 2016), improving our stress resilience in daily life, also in the presence of JI. Furthermore, viewed more generally, it would be beneficial to reduce JI among working parents. Earlier studies on the antecedents of JI show that, e.g., precarious/temporary employment, lower socioeconomic status, having children, organizational changes and poor communication regarding these changes predict increased JI (Kachi et al., 2018; Keim et al., 2014). Temporary employment contract was also associated with higher JI among working mothers in our study. Thus, working 
parents encountering these risk factors should be helped in organizations because eliminating the antecedents of JI among parents may prevent transmission of JI to their adolescent children.

\section{Recommendations for children and adolescents}

High self-esteem functions as a personal stress buffer in life adversities. Self-esteem can be cultivated in childhood and adolescence but tends to become more stable in adulthood (Rector \& Roger, 1997). As we found that adolescents' high self-esteem was protective against mothers' JI in relation to depressive symptoms, we recommend that children should receive various positive experiences in their daily living and social interactions, in daycare, at school, at home, and through hobbies, as such repeated positive experiences would boost self-esteem (see also Hong et al., 2016). For instance, enhancing pupils' self-esteem could be one valuable long-term target at schools. Educational interventions are also needed, focusing on increasing overall awareness that parents' negative work experiences, including JI, may also affect their children, and that boosting children's personal resources, e.g., self-esteem, may serve as a protective factor against parental job stress.

Acknowledgements This research is part of the STAIRWAY study and was supported by the Academy of Finland [grants 308 334, 266 851 and 294970]. The ideas and data presented in this article have not been previously disseminated.

\section{Compliance with Ethical Standards}

Conflict of Interest The authors declare no competing interests.

Ethical Approval This study was conducted in compliance with APA ethical standards. It was approved by the Ethics Committee of the University of Jyväskylä

Informed Consent Informed consent was obtained from all the participants of the study.

Publisher's note Springer Nature remains neutral with regard to jurisdictional claims in published maps and institutional affiliations.

Open Access This article is licensed under a Creative Commons Attribution 4.0 International License, which permits use, sharing, adaptation, distribution and reproduction in any medium or format, as long as you give appropriate credit to the original author(s) and the source, provide a link to the Creative Commons license, and indicate if changes were made. The images or other third party material in this article are included in the article's Creative Commons license, unless indicated otherwise in a credit line to the material. If material is not included in the article's Creative Commons license and your intended use is not permitted by statutory regulation or exceeds the permitted use, you will need to obtain permission directly from the copyright holder. To view a copy of this license, visit http://creativecommons. org/licenses/by/4.0/.

\section{References}

Barling, J., \& Mendelson, M. B. (1999). Parents' job insecurity affects children's grade performance through the indirect effects of beliefs in an unjust world and negative mood. Journal of Occupational Health Psychology, 4, 347-355.

Beck, A. T., Ward, C. H., Mendelson, M., Mock, J., \& Erbaugh, J. (1961). An inventory for measuring depression. Archives of General Psychiatry, 4, 561-571. https://doi.org/10.1001/a rchpsyc.1961.01710120031004.

Bennett, A., Bakker, A., \& Field, J. (2018). Recovery from workrelated effort: a meta-analysis. Journal of Organizational Behavior, 39, 262-275. https://doi.org/10.1002/job.2217.

Boden, J. M., Fergusson, D. M., \& Horwood, L. J. (2008). Does adolescent self-esteem predict later life outcomes? A test of the causal role of self-esteem. Development and Psychopathology, 20, 319-39. https://doi.org/10.1017/S0954579408000151.

Bor, W., Dean, A. J., Najman, J., \& Hayatbakhsh, R. (2014). Are child and adolescent mental health problems increasing in the $21 \mathrm{st}$ century? A systematic review. Australian and New Zealand Journal of Psychiatry, 48, 606-616. https://doi.org/10.1177/ 0004867414533834.

Chapman, R., Parkinson, M., \& Halligan, S. (2016). How do parentchild interactions predict and maintain depression in childhood and adolescence? A critical review of the literature. Adolescent Psychiatry, 6(2), 100-115. https://doi.org/10.2174/ 2210676606666160822101450.

Cheng, G. H. L., \& Chan, D. K. S. (2008). Who suffers more from job insecurity? A meta-analytic review. Applied psychology: An International Review, 57, 272-303. https://doi.org/10.1111/j. 1464-0597.2007.00312.x.

Conger, R. D., Conger, K. I., \& Martin, M. J. (2010). Socioeconomic status, family processes, and individual development. Journal of Marriage and the Family, 72(3), 685-704. https://doi.org/10. 1111/j.1741-3737.2010.00725.x.

Costello, E. J., Mustillo, S., Erkanli, A., Keeler, G., \& Angold, A. (2003). Prevalence and development of psychiatric disorders in childhood and adolescence. Archives of General Psychiatry, 60 (8), 837-844. https://doi.org/10.1001/archpsyc.60.8.837.

De Witte, H., Pienaar, J., \& De Cuyper, N. (2016). Review of 30 years of longitudinal studies on the association between job insecurity and health and well-being. Australian Psychologist, 51, 18-31. https://doi.org/10.1111/ap.12176.

Fletcher, J. (2008). Adolescent depression and educational attainment: results using sibling fixed effects. Health Economics, 17, 1215-1235. https://doi.org/10.1002/hec.1319.

Frone, M. R. (2003). Work-family balance. In J. C. Quick, \& L. E. Tetrick (Eds.), Handbook of occupational health psychology (pp. 143-162). American Psychological Association. https://doi.org/ 10.1037/10474-007.

Han, W.-J., \& Miller, D. P. (2009). Parental work schedules and adolescent depression. Health Sociology Review, 18(1), 36-49. https://doi.org/10.5172/hesr.18.1.36.

Hatfield, E., Cacioppo, J., \& Elliot, A. (1994). Emotion contagion. New York, NY: Cambridge University Press.

Hobfoll, S. (1989). Conservation of resources: A new attempt at conceptualizing stress. American Psychologist, 44, 513-524. https://doi.org/10.1037/0003-066X.44.3.513.

Hong, S. H., No, U. K., \& Jung, S. (2016). Meta-analysis about relationship between adolescents' suicidal ideation and protective factors. Survey Research, 17(1), 137-166. https://doi.org/10. 20997/SR.17.1.7.

Jiang, L. X., \& Lavaysse, L. M. (2018). Cognitive and affective job insecurity: a meta-analysis and a primary study. Journal of 
Management, 44(6), 2307-2342. https://doi.org/10.1177/ 0149206318773853.

Kachi, Y., Hashimoto, H., \& Eguchi, H. (2018). Gender differences in the effects of job insecurity on psychological distress in Japanese workers: a population-based panel study. International Archives of Occupational and Environmental Health, 91(8), 991-999. https://doi.org/10.1007/s00420-018-1338-z.

Keim, A., Landis, R., Pierce, C., \& Earnest, D. (2014). Why do employees worry about their jobs? A meta-analytic review of predictors of job insecurity. Journal of Occupational Health Psychology, 19(3), 269-290. https://doi.org/10.1037/a0036743.

Kim, T. J., \& von dem Knesebeck, O. (2016). Perceived job insecurity, unemployment and depressive symptoms: a systematic review and meta-analysis of prospective observational studies. International Archives of Occupations, Environment and Health, 89, 561-573. https://doi.org/10.1007/s00420-015-1107-1.

Kinnunen, U., Mauno, S., \& Siltaloppi, M. (2010). Job insecurity, recovery and well-being at work: recovery experiences as moderators. Economic and Industrial Democracy, 31, 179-194. https://doi.org/10.1177/0143831X09358366.

Klein, A., \& Moosbrugger, H. (2000). Maximum likelihood estimation of latent interaction effects with the LMS method. Psychometrika, 65, 457-474. https://doi.org/10.1007/BF02296338.

Lee, T. K., Wickrama, K. A., \& Simons, L. G. (2013). Chronic family economic hardship, family processes and progression of mental and physical health symptoms in adolescence. Journal of Youth and Adolescence, 42(6), 821-836. https://doi.org/10.1007/ s10964-012-9808-1.

Li, J., Johnson, S. E., Han, W.-J., Andrews, S., Kendall, G., Strazdins, L., \& Cockery, A. (2014). Parents' nonstandard work schedules and child well-being: a critical review of the literature. Journal of Primary Prevention, 35(1), 53-73. https://doi.org/10.1007/ s10935-013-0318-z.

Lim, V. K. G. \& Loo, G. L. (2003). Effects of parental job insecurity and parenting behaviors on youth's self-efficacy and work attitudes. Journal of Vocational Behavior, 63(1), 86-98. https://doi. org/10.1016/S0001-8791(02)00020-9.

MacKinnon, D., Lockwood, C., \& Williams, J. (2004). Confidence limits for the indirect effect: distribution of the product and resampling methods. Multivariate Behavioral Research, 39, 99-128. https://doi.org/10.1207/s15327906mbr3901_4.

Marsh, H., Hau, K., \& Wen, Z. (2004). In search of golden rules: comment on hypothesis-testing approaches to setting cutoff values for fit indexes and dangers in overgeneralizing $\mathrm{Hu}$ and Bentler's (1999) findings. Structural Equation Modeling: A Multidisciplinary Journal, 11, 320-341. https://doi.org/10.1207/ s15328007sem1103_2.

Mattila-Holappa, P. (2018). Mental health and labour market participation among young adults. Studies in Social Security and Health, 152. The Social Insurance Institution of Finland. http:// urn.fi/URN:NBN:fi-fe2018082934286

Mauno, S., Cheng, T., \& Lim, V. (2017). The far-reaching consequences of job insecurity: a review on family-related outcomes.
Marriage and Family Review, 53, 717-743. https://doi.org/10. 1080/01494929.2017.1283382.

Mauno, S., \& Kinnunen, U. (1999). The effects of job stressors on marital satisfaction in Finnish dual-earner couples. Journal of Organizational Behavior, 19, 1-18. https://doi.org/10.1002/ (SICI)1099-1379(199911)20:6<879::AID-JOB982>3.0.CO;2-2.

McLaughlin, K. A., Breslau, J., Green, J. G., Lakoma, M. D., Sampson, N. A., Zaslavsky, A. M., \& Kessler, R. C. (2011). Childhood socio-economic status and the onset, persistence, and severity of DSM-IV mental disorders in a US national sample. Social Science Medicine, 73(7), 1088-1096. https://doi.org/10. 1016/j.socscimed.2011.06.011.

Meijman, T. E., \& Mulder, G. (1998). Psychological aspects of workload. In P. J. D. Drenth, \& H. Thierry (Eds.), Handbook of work and organizational psychology: Vol. 2. Work Psychology (pp. 5-33). Psychology Press.

Muthén, L.K., \& Muthén, B.O. (1998-2017). Mplus User's Guide (8th ed.). Muthén \& Muthén.

Niks, I., Gevers, J., De Jonge, J., \& Houtman, I. (2016). The relation between off-job recovery and job resources: person-level differences and day-level dynamics. European Journal of Work and Organizational Psychology, 25, 226-238. https://doi.org/10. 1080/1359432X.2015.1042459.

Official Statistics of Finland (2018a) Educational structure of population. Retrieved from Statistics Finland website: http://www.stat. fi/til/vkour/2014/vkour_2014_2015-11-05_tie_001_en.html

Official Statistics of Finland (2018b). Families. Appendix Table 3: Families with underage children by type in 1950-2014. Retrieved from Statistics Finland website: http://www.stat.fi/til/perh/2014/ perh_2014_2015-05-28_tau_003_en.html.

Rector, N. A., \& Roger, D. (1997). The stress buffering effects of selfesteem. Personality and Individual Differences, 23(5), 799-808. https://doi.org/10.1016/S0191-8869(97)00095-0.

Rosenberg, M. (1965). Society and the adolescent self-image. Princeton University Press.

Rosenberg, M. (1979). Conceiving the self. Basic Books.

Salokangas, R. K. R., Poutanen, O., \& Stengård, E. (1995). Screening for depression in primary care. Development and validation of the Depression Scale, a screening instrument for depression. Acta Psychiatrica Scandinavica, 92, 10-16. https://doi.org/10.1111/j. 1600-0447.1995.tb09536.x.

Taewoo, N. (2019). Technology usage, expected job sustainability and perceived job insecurity. Technological Forecasting \& Social Change, 138, 155-165. https://doi.org/10.1016/j.techfore.2018. 08.017 .

Vander Elst, T., De Witte, H., \& De Cuyper, N. (2014). The Job Insecurity Scale: A psychometric evaluation across five European countries. European Journal of Work and Organizational Psychology, 23, 364-380. https://doi.org/10.1007/978-94-017-9798-6_7.

Zhao, X., Lim, K. G. V., \& Teo, T. S. H. (2012). The long arm of job insecurity: Its impact on career-specific parenting behaviors and youths' career self-efficacy. Journal of Vocational Behavior, 80, 619-628. https://doi.org/10.1016/j.jvb.2012.01.018. 